\title{
Chitosan-Coated-PLGA Nanoparticles Enhance the Antitumor and Antimigration Activity of Stattic - A STAT3 Dimerization Blocker
}

\begin{abstract}
Stephanie Sally Fong, ',* Yiing Yee Foo, ${ }^{1, *}$ Wen Shang Saw, (D) ${ }^{2}$ Bey Fen Leo, ${ }^{3}$ Yin Yin Teo, ${ }^{4}$ Ivy Chung, 'Boon Tong Goh, ${ }^{5}$ Misni Misran, ${ }^{4}$ Toyoko Imae, ${ }^{6}$ Chia-Ching Chang, (iD) ${ }^{7-11}$ Lip Yong Chung, (iD) ${ }^{2}$ Lik Voon Kiew (iD)

'Department of Pharmacology, Faculty of Medicine, University of Malaya, Kuala Lumpur, 50603, Malaysia; ${ }^{2}$ Department of Pharmaceutical Chemistry, Faculty of Pharmacy, University of Malaya, Kuala Lumpur, 50603, Malaysia; ${ }^{3}$ Faculty of Medicine, University of Malaya, Kuala Lumpur. 50603, Malaysia; ${ }^{4}$ Department of Chemistry, Faculty of Science, University of Malaya, Kuala Lumpur, 50603, Malaysia; ' ${ }^{5}$ ow Dimensional Materials Research Center, Department of Physics, Faculty of Science, University of Malaya, Kuala Lumpur, 50603, Malaysia; ${ }^{6}$ Graduate Institute of Applied Science and Technology, National Taiwan University of Science and Technology, Taipei, 10607, Taiwan; ${ }^{7}$ Department of Biological Science and Technology, College of Biological Science and Technology, National Yang Ming Chiao Tung University, Hsinchu, 30068, Taiwan; ${ }^{8}$ Center for Intelligent Drug Systems and Smart Bio-Devices (IDS ${ }^{2} \mathrm{~B}$ ), National Yang Ming Chiao Tung University, Hsinchu, 30050, Taiwan; ' ${ }^{\circ}$ Department of Electrophysics, National Yang Ming Chiao Tung University, Hsinchu, 30010, Taiwan; ${ }^{10}$ Institute of Physics, Academia Sinica, Nankang, Taipei, Taiwan; " Taiwan-Malaysia Semiconductor and Biomedical Oversea Science and Technology Innovation Center, National Yang Ming Chiao Tung University, Hsinchu, 300I0, Taiwan
\end{abstract}

*These authors contributed equally to this work

Correspondence: Lik Voon Kiew

Department of Pharmacology, Faculty of

Medicine, University of Malaya, Kuala Lumpur,

50603, Malaysia

Tel +603-79675720

Email Ivkiew@um.edu.my

Chia-Ching Chang

Department of Biological Science and

Technology, College of Biological Science and

Technology, National Yang Ming Chiao Tung

University, Hsinchu, 30068, Taiwan

Tel +886-3-57I3I633

Email ccchang0I@nycu.edu.tw
Purpose: The use of nanocarriers to improve the delivery and efficacy of antimetastatic agents is less explored when compared to cytotoxic agents. This study reports the entrapment of an antimetastatic Signal Transducer and Activator of Transcription 3 (STAT3) dimerization blocker, Stattic (S) into a chitosan-coated-poly(lactic-co-glycolic acid) (C-PLGA) nanocarrier and the improvement on the drug's physicochemical, in vitro and in vivo antimetastatic properties post entrapment.

Methods: In vitro, physicochemical properties of the Stattic-entrapped C-PLGA nanoparticles (S@C-PLGA) and Stattic-entrapped PLGA nanoparticles (S@PLGA, control) in terms of size, zeta potential, polydispersity index, drug loading, entrapment efficiency, Stattic release in different medium and cytotoxicity were firstly evaluated. The in vitro antimigration properties of the nanoparticles on breast cancer cell lines were then studied by Scratch assay and Transwell assay. Study on the in vivo antitumor efficacy and antimetastatic properties of S@C-PLGA compared to Stattic were then performed on 4T1 tumor bearing mice.

Results: The S@C-PLGA nanoparticles $(141.8 \pm 2.3 \mathrm{~nm})$ was hemocompatible and exhibited low Stattic release (12\%) in plasma. S@C-PLGA also exhibited enhanced in vitro anticell migration potency (by $>10$-fold in MDA-MB-231 and 5-fold in 4T1 cells) and in vivo tumor growth suppression (by 33.6\%) in $4 \mathrm{~T} 1$ murine metastatic mammary tumor bearing mice when compared to that of the Stattic-treated group. Interestingly, the number of lung and liver metastatic foci was found to reduce by $50 \%$ and $56.6 \%$, respectively, and the average size of the lung metastatic foci was reduced by $75.4 \%$ in $4 \mathrm{~T} 1$ tumor-bearing mice treated with S@C-PLGA compared to Stattic-treated group $(\mathrm{p}<0.001)$.

Conclusion: These findings suggest the usage of C-PLGA nanocarrier to improve the delivery and efficacy of antimetastatic agents, such as Stattic, in cancer therapy.

Keywords: nanocarrier, STAT3 protein, breast cancer, metastasis, chitosan-coating

\section{Introduction}

Tumor metastasis contributes significantly to cancer deaths, and effective delivery of antimetastatic agents to the tumor may assist in suppressing the progression of the disease. ${ }^{1}$ Although nanocarriers have been frequently reported to improve the delivery and anticancer efficacy of cytotoxic agents, ${ }^{2-6}$ relatively few investigations of a similar nature have been made on the nano-delivery of antimetastatic agents.

The Signal Transducer and Activator of Transcription 3 (STAT3) signaling pathway has been identified as a key contributor to the development of metastatic traits in tumors, and STAT3 inhibitors have been developed as a potential lead for antimetastasis. ${ }^{7,8}$ Among these STAT3 inhibitors, Stattic, a small molecular inhibitor, was reported to 


\section{Graphical Abstract}
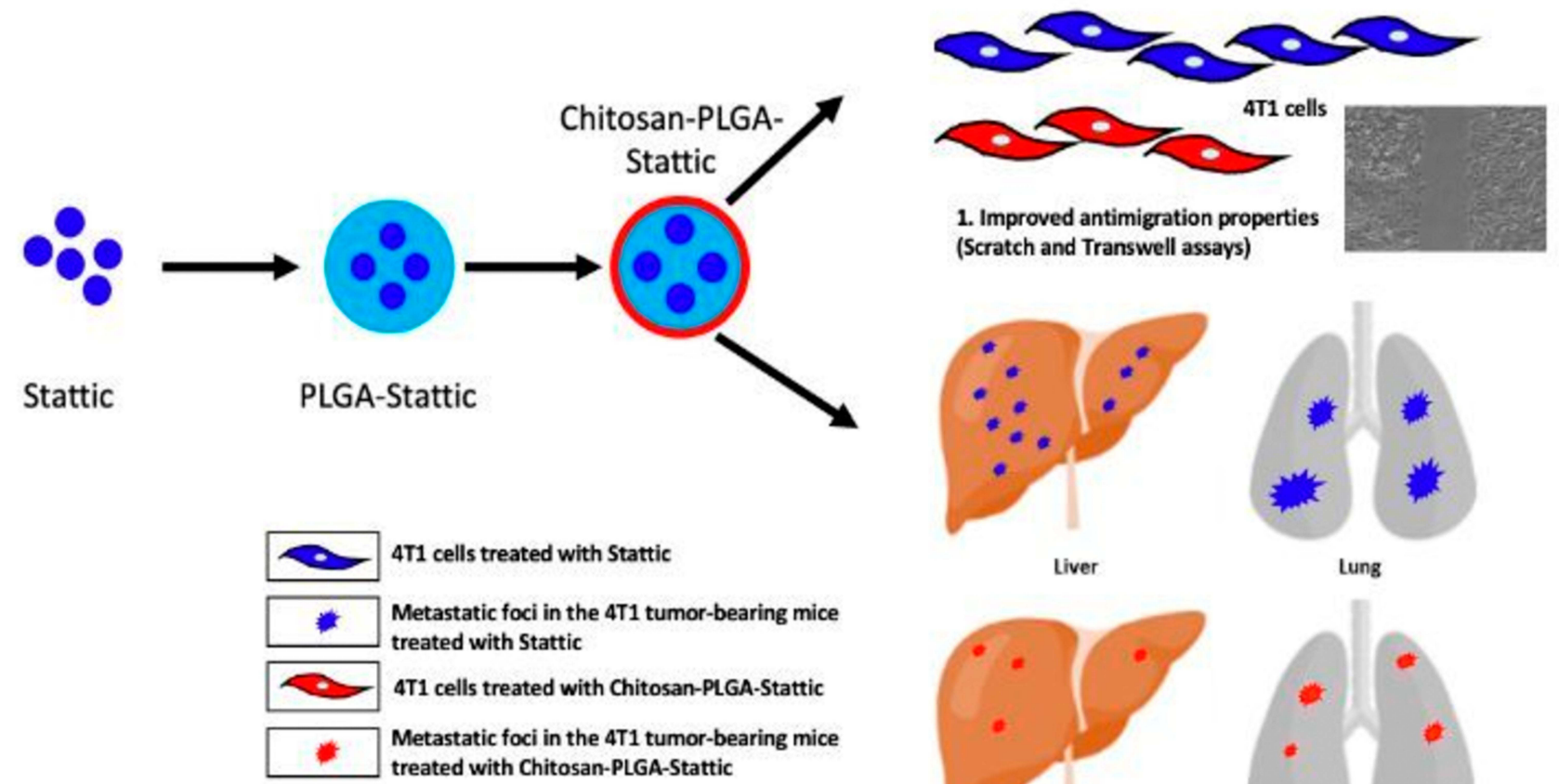

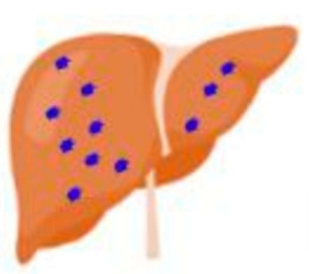

Liver

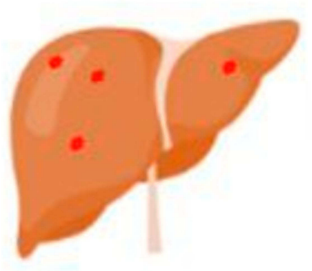

2. $4 \mathrm{~T} 1$ murine mammary tumor model:

- Reduced the number of liver metastatic foci

- Reduced the average size of lung metastatic focl selectively inhibit phosphorylation, activation, dimerization, and nuclear translocation of STAT3., ${ }^{9,10}$ Stattic specifically targets the SH2 domain of the STAT3 protein, but not other STAT proteins, and hence it produces improved pharmacological selectivity. ${ }^{7,11}$ Stattic inhibition of STAT3 signaling has been reported to induce apoptosis in STAT3-dependent cancer cells (eg, MDA-MB-231 and MDA-MB-435S) and reduce tumor size in murine breast cancer (HC-11). ${ }^{10,12}$ However, a relatively high concentration of Stattic (up to $20 \mu \mathrm{M}$ ) is required to suppress STAT3 activity in cancer cells in vitro. ${ }^{7,11}$ The low in vitro potency and poor water solubility of Stattic have impeded its clinical use for antimetastasis.

Entrapment of drugs into biocompatible nanocarriers may increase their apparent solubility and stability in the blood, prevent premature clearance by the reticuloendothelial system, prolong their circulation time, and reduce nonselective drug toxicity. ${ }^{4,13,14}$ The aberrant traits of mature tumor tissues, such as leaky vasculature and poor lymphatic drainage, may promote the accumulation of nanocarrierdrug complexes through the enhanced permeability and retention (EPR) effect. ${ }^{15,16}$ In our earlier studies, chitosan coating on the surfaces of photosensitizer-loaded PLGA nanoparticles (NPs) reduced the burst and premature release of drugs and decreased the macrophage uptake of NPs. Additionally, these chitosan-coated PLGA-photosensitizer NPs also exhibited elevated in vitro cancer cell uptake and phototoxicity, as well as in vivo reduction in reticuloendothelial tissue uptake, enhanced tumor accumulation, and antitumor efficacy compared with uncoated PLGAphotosensitizer NPs. ${ }^{17}$ Thus, we entrapped Stattic in a chitosan-coated poly (lactic-co-glycolic acid) [C-PLGA] nanocarrier and investigated the improvements to its in vitro and in vivo antitumor and antimetastatic activities and efficacies for potential applications in cancer therapy.

\section{Materials and Methods \\ Materials}

Poly(D, L-lactic-co-glycolic-acid) [50:50, MW 38,00054,000], poly (vinyl alcohol) [PVA, MW 30,000-70,000] and 3-(4, 5-dimethylthiazol-2-yl-2, 5-diphenyl tetrazolium bromide (MTT) were procured from Sigma-Aldrich (St. Louis, MO, USA). Stattic was purchased from 
MedChem Express ( $>98 \%$ purity, Monmouth Junction, NJ, USA). Chitosan powder with a molecular weight of $25 \mathrm{kDa}$ was supplied by the Department of Chemistry, University of Malaya, Malaysia. ${ }^{18}$ Chemicals such as hydrochloric acid, acetonitrile, ethyl acetate, dichloromethane, and ethanol were purchased from Merck (Darmstadt, Hesse, Germany). 4T1 and MDA-MB-231 cells were purchased from ATCC (Manassas, VA, USA), Dulbecco's modified Eagle medium (DMEM), Roswell Park Memorial Institute (RPMI) 1640 medium, fetal bovine serum (FBS), trypsin, and penicillin-streptomycin were acquired from Gibco $^{\circledR}$ (Grand Island, NY, USA).

\section{Methods}

Preparation of PLGA, S@PLGA, and S@C-PLGA NPs

Both blank PLGA and S@PLGA NPs were prepared via nanoprecipitation methods, as previously described with minor modifications. ${ }^{19}$ Briefly, $12 \mathrm{mg}$ Stattic and $60 \mathrm{mg}$ PLGA were mixed in acetonitrile and added dropwise to a solution containing $1 \% \mathrm{w} / \mathrm{v}$ of surfactant, poly (vinyl alcohol), to lower the surface tension of nanoparticle surfaces and prevent particle agglomeration, as illustrated in Scheme 1, followed by stirring overnight after homogenization and sonication. The resulting mixture was then centrifuged at $2700 \times \mathrm{g}$ for $30 \mathrm{~min}$, followed by ultracentrifugation at $102,000 \times \mathrm{g}$ at $4{ }^{\circ} \mathrm{C}$ for $30 \mathrm{~min}$ to obtain S@PLGA NPs (Optima LE-80K, Beckman Coulter, Brea, CA, USA). The pellet was resuspended in deionized water and lyophilized. The lyophilized nanoparticles were weighed and stored at $4{ }^{\circ} \mathrm{C}$ until further use. The blank PLGA NPs were prepared in the same way as described above without the addition of Stattic. For the preparation of S@C-PLGA, S@PLGA NPs were added to a chitosan solution $(2 \% \mathrm{w} / \mathrm{v})$, stirred overnight, and kept at $4{ }^{\circ} \mathrm{C}$ until use.

\section{Characterization of Blank PLGA, S@PLGA, and S@C-PLGA}

The particle size, zeta potential, and polydispersity index (PDI) of blank PLGA, S@PLGA, and
S@C-PLGA NPs were measured using a Malvern Zetasizer Nano S90 (Malvern Instruments Ltd., UK). The samples were prepared by diluting the nanoparticle dispersion in deionized water and sonicating for 30 $\mathrm{s}$ before measurement. The size and zeta potential of the nanoparticles with different concentrations of Stattic loaded were also measured after incubation in cell culture medium for $24 \mathrm{~h}$.

For transmission electron microscope (TEM) sample preparation, the diluted sample was dropped onto a copper grid, stained with $0.25 \%$ uranyl acid replacement (UAR) solution for $60 \mathrm{~s}$, and allowed to dry. The morphology of the particles was observed using a Carl Zeiss Libra 120 PLUS TEM (Oberkochen, Germany) at $120 \mathrm{kV}$.

\section{Loading and Entrapment Efficiency of Stattic}

To evaluate the percentage of loading and entrapment efficiency of Stattic in the NPs, $1 \mathrm{mg}$ of lyophilized S@PLGA NPs was dissolved in $1 \mathrm{~mL}$ of acetonitrile for extraction. The solution was incubated at room temperature overnight, followed by centrifugation at $9300 \times \mathrm{g}$ for $10 \mathrm{~min}$. The weight of Stattic in the NPs was determined by first measuring the absorbance of the supernatant $(1 \mathrm{~mL})$ at $318.5 \mathrm{~nm}$ using an ultraviolet-visible (UV-Vis) spectrophotometer (PerkinElmer, Waltham, MA, USA). Thereafter, the concentration of Stattic in the supernatant was determined using a Stattic concentration standard curve (built by measuring the absorbance of acetonitrile solutions containing escalating concentrations of Stattic; Figure S1). Based on the concentration value obtained, the weight of Stattic in NPs $(1 \mathrm{mg})$ was calculated.

The loading and entrapment efficiencies were then estimated using Equations (1) and (2), respectively:

Stattic loading $(\% \mathrm{w} / \mathrm{w})=\frac{\text { weight of Stattic in NPs }}{\mathrm{M}_{\mathrm{NP}}} \times 100$

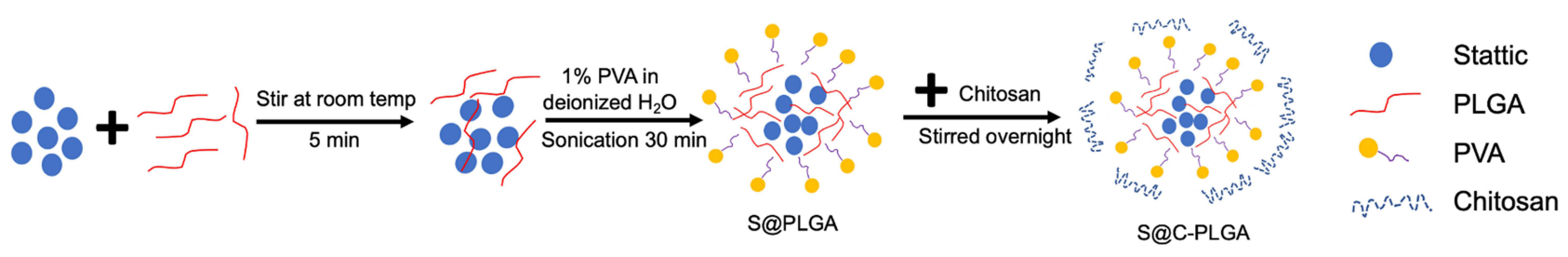

Scheme I The synthesis of S@PLGA and S@C-PLGA NPs. 


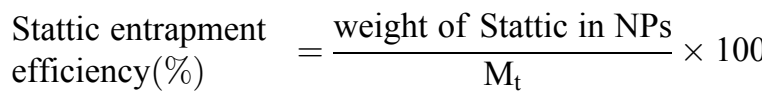

where $\mathrm{M}_{\mathrm{NP}}$ is the weight of lyophilized NPs used for Stattic loading estimation $(1 \mathrm{mg}$, dissolved in $1 \mathrm{~mL}$ acetonitrile), $M_{t}$ is the weight of Stattic used during the preparation of a batch of NP.

\section{Stattic Releasing Profile in Phosphate Buffer Saline and Plasma}

The in vitro release of Stattic in phosphate buffer solution (PBS) at $\mathrm{pH} 7.4$ and $\mathrm{pH} \mathrm{4.8,} \mathrm{and} \mathrm{in} \mathrm{human} \mathrm{plasma} \mathrm{was}$ assessed based on previously described protocols ${ }^{17,20}$ with modifications. S@PLGA NPs and S@C-PLGA NPs containing an equivalent amount of Stattic $(50 \mu \mathrm{g} / \mathrm{mL})$ were suspended in PBS or plasma $(25 \mathrm{~mL})$. The solution was then aliquoted into multiple centrifuge tubes and placed on a Thermo shaker at $37{ }^{\circ} \mathrm{C}$ and agitated at $200 \mathrm{rpm}$. A tube was removed from the shaker at different time points $(0,0.5,1,2,4,8,12,24$, and $48 \mathrm{~h})$ and centrifuged at $9300 \times \mathrm{g}$ for $10 \mathrm{~min}$. The absorbance of the supernatant was recorded at $318.5 \mathrm{~nm}$ using a UV-Vis spectrophotometer to quantify the amount of released Stattic. The percentage of Stattic release in PBS or plasma was calculated using Equation (3) as follows:

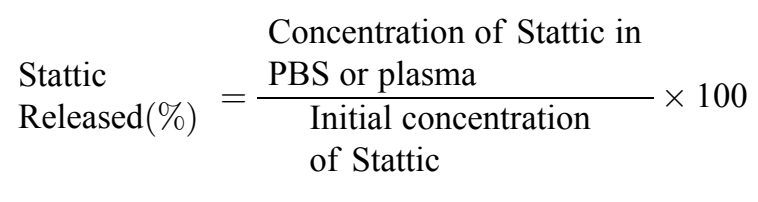

\section{Scratch Assay}

Scratch assay was performed based on a previously published protocol to evaluate cell migration. ${ }^{21}$ Briefly, a density of 100,000 cells per well of breast cancer cell lines (4T1 and MDA-MB-231) were seeded in 24-well plates and incubated overnight to reach confluency. A pipette tip $(10 \mu \mathrm{L})$ was used to make a straight scratch on each well. The media was then removed and washed twice with PBS before treatment with different concentrations of Stattic, S@PLGA NPs, and S@C-PLGA NPs. The cells were observed under an inverted microscope (Nikon Eclipse Ti, Nikon Corp., Minato, Tokyo, Japan) for $24 \mathrm{~h}$, and the images were captured at $10 \times$ magnification. The rate of migration was determined from the slope of the percentage migration over the time plot.

\section{Transwell Assay}

Transwell assay (migration) was performed according to a previously described protocol ${ }^{22}$ with minor modifications. Briefly, the cells $(250,000$ cells/well $)$ were seeded in the upper chamber of the cell insert in serum-free medium with an $8 \mu \mathrm{m}$ pore size. Different concentrations of the samples were added to the upper chamber. Media containing $10 \%$ FBS were added to the lower chamber to create a chemoattractant gradient. The plates were incubated for $12 \mathrm{~h}$. At the end of the incubation, the medium was removed from the cell insert and washed twice with PBS. The cells were fixed in a formalin solution $(3.7 \%$ formaldehyde) for $2 \mathrm{~min}$. The solution was removed and the cells were washed twice with PBS. Methanol was added to the insert for $20 \mathrm{~min}$ to permeabilize the cells. The cells were then stained with $0.5 \%$ crystal violet for $15 \mathrm{~min}$. The excess crystal violet stain was removed by washing with PBS, and non-migrated cells in the upper chamber were scraped off using a cotton swab. Images of the cells were captured using an inverted microscope, and the number of migrated cells was calculated using the ImageJ software. ${ }^{22}$

\section{In vitro Cytotoxicity}

To determine the cytotoxicity of Stattic, S@PLGA, and S@C-PLGA NPs on murine breast cancer cells (4T1), 3-(4, 5 dimethylthiazol-2-yl-2, 5-diphenyl tetrazolium bromide (MTT) assay was performed as described by Mosmann (1983). Briefly, $100 \mu \mathrm{L}$ of cells at a density of 10,000 cells per well in 96 well plates were seeded and incubated overnight at $37{ }^{\circ} \mathrm{C}$ and $5 \% \mathrm{CO}_{2}$ in a $\mathrm{CO}_{2}$ incubator to allow cell attachment. Cells were treated with different concentrations of Stattic, S@PLGA, and S@C-PLGA NPs and further incubated for $24 \mathrm{~h}$. After incubation, MTT reagent was added to the wells and incubated for $4 \mathrm{~h}$ in the dark. Subsequently, the medium was aspirated and $100 \mu \mathrm{L}$ of dimethyl sulfoxide (DMSO) was added to dissolve the purple formazan crystals. Formazan accumulation showed mitochondrial activity in the viable cells. The percentage of cell viability was calculated based on Equation (4) using the absorbance values obtained at $570 \mathrm{~nm}$ :

Percentage of

cell viability $(\%)=\frac{\text { Absorbance }_{\text {control }}-\text { Absorbance }_{\text {sample }}}{\text { Absorbance }_{\text {control }}} \times 100$ 


\section{In vivo Studies}

Female immunocompetent wild-type BALB/c mice (body weight of 18-20 g; 8-10 weeks old) were supplied by and maintained in the Animal Experimental Unit, Faculty of Medicine, University of Malaya for in vivo studies. The mice were kept in a controlled environment with a 12-h light-dark cycle and free access to food and water. All animal experiments were carried out following the protocols and ethics approved by the Faculty of Medicine Institutional Animal Care and Use Committee of the University of Malaya (FOM IACUC; Ethics Reference no. 2020-210206/PHAR/R/SSF).

\section{In vivo Toxicity Profiles of Stattic and S@C-PLGA} NPs

Stattic and S@C-PLGA NPs were administered intravenously (via tail vein) to the mice at a dosage of 12 or 24 mg Stattic equivalent $/ \mathrm{kg}$. Stattic and S@C-PLGA NPs were prepared in saline solution. The 21-day toxicity observations (Berlin's test) were carried out by monitoring and recording symptoms, including behavioral changes, ruffled fur, inactivity, and loss of body weight. ${ }^{17}$

\section{In vivo Antitumor Efficacy Studies in 4TI Tumor-Bearing Mice}

The 4T1 tumor model was previously reported as an optimal experimental animal model for the study of mammary tumor metastasis, as the tumor growth and metastatic spread features of the $4 \mathrm{~T} 1$ tumor in BALB/c mice closely mimic those observed in human metastatic breast cancer. ${ }^{23}$ Hence, in this study, 4T1 tumors were induced in female BALB/c mice according to a previously described method. ${ }^{17}$ The $4 \mathrm{~T} 1$ murine breast cancer cells $\left(5 \times 10^{5}\right.$ cells suspended in $0.1 \mathrm{~mL}$ of RPMI medium) were injected into the mammary fat pads of BALB/c mice after their fur was shaved. When the tumor reached an average volume of $70 \mathrm{~mm}^{3}$, the mice were intravenously injected via the tail vein with saline, Stattic, and S@C-PLGA at $24 \mathrm{mg}$ Stattic equivalent $/ \mathrm{kg}$ on 0th, 3rd, 6th, and 9th days. All the samples were prepared in saline to achieve an injection volume of $0.2 \mathrm{~mL}$. The weight of the mice was recorded daily. Tumor volumes were also measured daily using a caliper and calculated according to Equation (5) as follows:

$$
\text { Tumor volume }\left(\mathrm{mm}^{3}\right)=\frac{\mathrm{L} \times \mathrm{W}^{2}}{2}
$$

where $\mathrm{L}$ is the longest dimension and $\mathrm{W}$ is the shortest dimension. ${ }^{24,25}$ The area under the curve (AUC) for each group was determined using GraphPad Prism 8.3.0. For ethical reasons, the tumor volume was not allowed to exceed $1000 \mathrm{~mm}^{3}$ throughout the study.

\section{Histopathology Examination}

The major organs of the mice were harvested after 14 days of treatment and preserved in 4\% neutral-buffered formalin solution prior to tissue processing and staining. Sections of paraffin-embedded tissues were prepared and stained with hematoxylin and eosin. The slides were then viewed under a light microscope with a $40 \times$ objective (Nikon Eclipse E200, Nikon Corp., Minato, Tokyo, Japan), and the number of metastatic foci was counted; the area of metastatic foci for every slide was measured using the Image J software (NIH, Maryland, USA).

\section{Statistical Analysis}

The experiments were carried out in triplicate, and the results are expressed as mean \pm standard deviation (SD). Analysis of variance (ANOVA) for statistical differences between the groups was analyzed using GraphPad Prism 8.3.0 (GraphPad Software, San Diego, USA). Statistical significance was set at $\mathrm{p}<0.05$.

\section{Results and Discussion Physicochemical Characteristics and Biocompatibility of Nanoparticles}

S@PLGA and S@C-PLGA NPs were prepared at a yield of $86.3 \% \pm 2.2 \%$ and $87.1 \% \pm 1.2 \%$, respectively, which was similar to the blank PLGA NPs $(85.8 \% \pm 2.4 \%)$ (Table 1). Stattic loading was approximately $12 \%$ and the

Table I Characterization of Nanoparticles in Deionized Water

\begin{tabular}{|l|l|l|l|l|l|l|}
\hline Nanoparticles & Size (nm) & PDI & Zeta Potential (mV) & Yield (\%) & Loading Capacity (\%) & Entrapment Efficiency (\%) \\
\hline Blank PLGA & $122.3 \pm 3.6$ & $0.18 \pm 0.0 \mathrm{I}$ & $-16.6 \pm 2.2$ & $85.8 \pm 2.4$ & NA & NA \\
S@PLGA & $133.9 \pm 2.1$ & $0.19 \pm 0.0 \mathrm{I}$ & $-1.9 \pm 0.3$ & $86.3 \pm 2.2$ & $12.1 \pm 1.9$ & $50.3 \pm 2.0$ \\
S@C-PLGA & $141.8 \pm 2.3$ & $0.19 \pm 0.0 \mathrm{I}$ & $4.1 \pm 0.6$ & $87.1 \pm 1.2$ & $12.4 \pm 2.8$ & $54.2 \pm 3.5$ \\
\hline
\end{tabular}

Note: Data represent mean \pm SD from three independent experiments.

Abbreviations: NA, not applicable; PDI, polydispersity index. 
entrapment efficiencies in PLGA and C-PLGA NPs were $50 \%$ and $54 \%$, respectively.

The average diameter of S@PLGA NPs increased by $5.9 \%$ (from $133.9 \pm 2.1$ to $141.8 \pm 2.3 \mathrm{~nm}$ ) upon coating with chitosan, as determined by dynamic light scattering (DLS). TEM images showed that the blank PLGA, S@PLGA, and S@C-PLGA NPs (Figure 1A-C) were spherical with diameters ranging between 70 and $110 \mathrm{~nm}$ (Figure 1D). The chitosan coating on S@C-PLGA NPs is shown in Figure 1C (inset). The average diameter of the NPs determined using TEM is generally less than the values determined by DLS because DLS measures NPs' hydrodynamic size, which includes the hydration layer. ${ }^{26,27}$ Both S@PLGA and S@C-PLGA NPs have average sizes $<150$ nm, which are optimal for systemic administration and EPR effect-based accumulation in mature solid tumors. ${ }^{28}$ The zeta potential of S@PLGA NPs changed from $-1.9 \pm 0.3$ to +4.1 $\pm 0.6 \mathrm{mV}$ post chitosan coating, as summarized in Table 1 , presumably owing to the protonation of chitosan amines. The near-neutral zeta potential value may be able to minimize opsonization by van der Waals and ionic interactions. ${ }^{17}$ However, the chitosan coating did not significantly affect the PDI, yield, Stattic loading capacity, or entrapment efficiency.

All of the NPs synthesized (PLGA, S@PLGA, or S@C-PLGA) did not induce red blood cell aggregation (Figure S2) and caused $<5 \%$ hemolysis at the highest concentration tested (200 $\mu \mathrm{M}$ NPs; Figure S3), indicating good hemocompatibility. ${ }^{29}$

\section{Stattic Releasing Profile in PBS and Plasma}

Stattic entrapped in the C-PLGA NPs compared with noncoated PLGA NPs exhibited more favorable stability and lower drug release profiles in both PBS and plasma (Figure 2A and B). The maximum Stattic released from the S@C-PLGA in PBS at pH 7.4 (0.5\%) and $4.8(4.6 \%)$
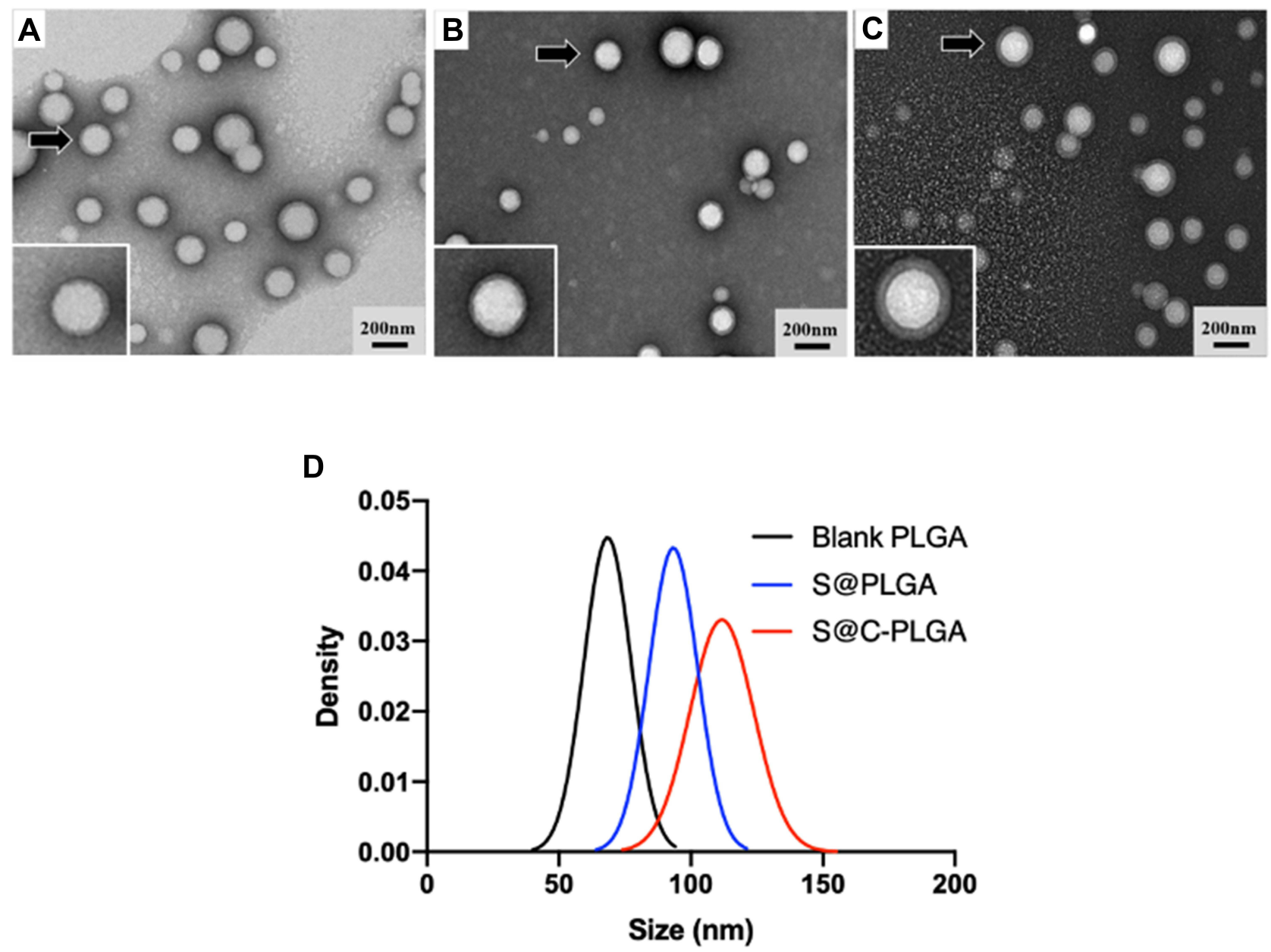

Figure I Evaluation of nanoparticles in term of size. Transmission electron micrographs of blank PLGA NPs (A), S@PLGA NPs (B) and S@C-PLGA NPs (C). Insets show a single particle denoted by arrow. (D) The normalized size distributions of nanoparticles obtained from the analysis of TEM images ( $n=100)$ that were quantified using Imagej software version 2.0 . 


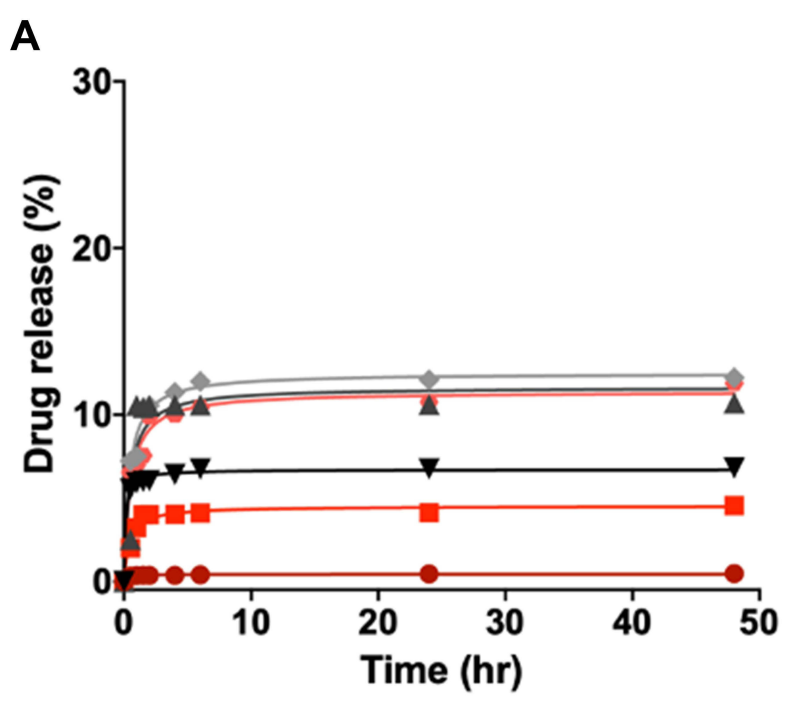

7 S@PLGA pH 7.4 S@C-PLGA pH 7.4
B

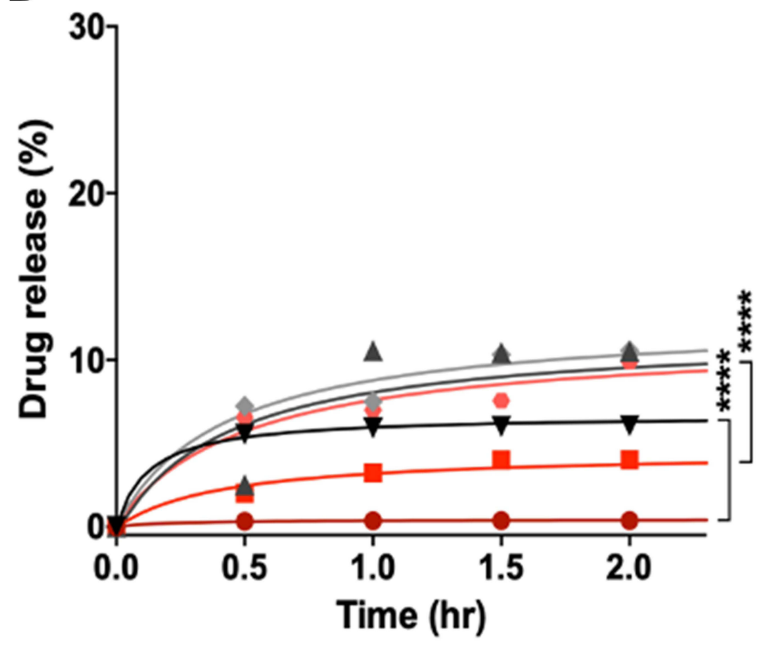

- S@PLGA Plasma

- S@C-PLGA Plasma

Figure 2 Stattic released in PBS and plasma. Cumulative percentage of Stattic released from S@PLGA NPs and S@C-PLGA NPs in different solution at 0-48 h (A) and 0-2 h (B). Data represent mean \pm SEM from three independent experiments; *****ndicate $\mathrm{p}<0.000 \mathrm{I}$, as assessed by two-way ANOVA with Tukey's post-hoc test.

was lower than that from the S@PLGA $(6.8 \%$ at pH 7.4, $10.7 \%$ at $\mathrm{pH} 4.8$ ) (Figure 2B). This suggests that chitosan coating reduced the release of Stattic from the nanocarrier and maintained the stability of S@C-PLGA in PBS. ${ }^{30}$

In plasma at pH 7.4, both S@C-PLGA NPs and S@PLGA NPs exhibited similar drug release patterns, where $\sim 12 \%$ of Stattic was released rapidly within $2 \mathrm{~h}$. This indicated rapid diffusion of Stattic from the polymeric matrix to the plasma protein, likely owing to affinity of Stattic towards albumin molecules. ${ }^{31}$ A similar observation has been reported previously, where Stattic entrapped within a synthetic polymeric micellar carrier $(\mathrm{P} 71 \mathrm{D} 3)^{32}$ was rapidly released when the drug-carriercomplexes were incubated in an albumin solution. ${ }^{33}$ The Stattic burst release from S@C-PLGA NPs in this study was considerably low $(<15 \%)$, suggesting the suitability of C-PLGA NPs as the delivery vehicles for Stattic and antimetastatic drugs with a similar chemical structure.

\section{Effects of Stattic, S@C-PLGA, and S@PLGA on Cytotoxicity and Cell Migration}

The cytotoxicity of Stattic, S@PLGA, and S@C-PLGA towards 4T1 breast cancer cell lines was assessed by the MTT assay (Figure S4). Stattic entrapped in PLGA and C-PLGA NPs exhibited higher cytotoxicity in metastatic
$4 \mathrm{~T} 1$ cells, with $\mathrm{IC}_{50} 44 \%$ and $45 \%$ lower than Stattic, respectively. The observed cytotoxicity was likely ascribed to the inhibition of STAT3 signaling that led to apoptosis of STAT3-dependent cancer cells through cleavage of caspase-3 poly (ADP-ribose) and polymerase (PARP). ${ }^{9,10}$ The enhancement of cytotoxicity of Stattic entrapped in nanocarriers may be due to endocytosis, which allows more Stattic to be taken up into the cells. ${ }^{34,35}$ We have previously demonstrated that BODIPY entrapped in PLGA and C-PLGA NPs were taken up into 4T1 cells via endocytosis. $^{17}$

Cancer cell migration is an essential hallmark of cancer metastasis. ${ }^{36}$ In this study, the changes in the antimigration properties of Stattic before and after its entrapment into the C-PLGA NPs (ie, Stattic vs S@C-PLGA) were assessed using human metastatic MDA-MB-231 cells and murine metastatic 4T1 cells. $^{20,37}$ Stattic entrapped in the C-PLGA nanocarrier exhibited better in vitro antimigration effects than S@PLGA and Stattic, whereby S@C-PLGA at $1 \mu \mathrm{M}$ Stattic equivalent concentration suppressed the migration of MDA-MB-231 (Figure 3A) and 4T1 (Figure 3B) cells by $65.1 \%$ and $47.3 \%$, respectively (Figure 3C). In contrast, similar levels of antimigration effects were only achieved when MDA-MB and 4T1 cells were exposed to $>10$ and $5 \mu \mathrm{M}$ Stattic (Figure S5), respectively, which were $>10$ - and 5-fold higher than that of S@C-PLGA 
A

MDA-MB-231
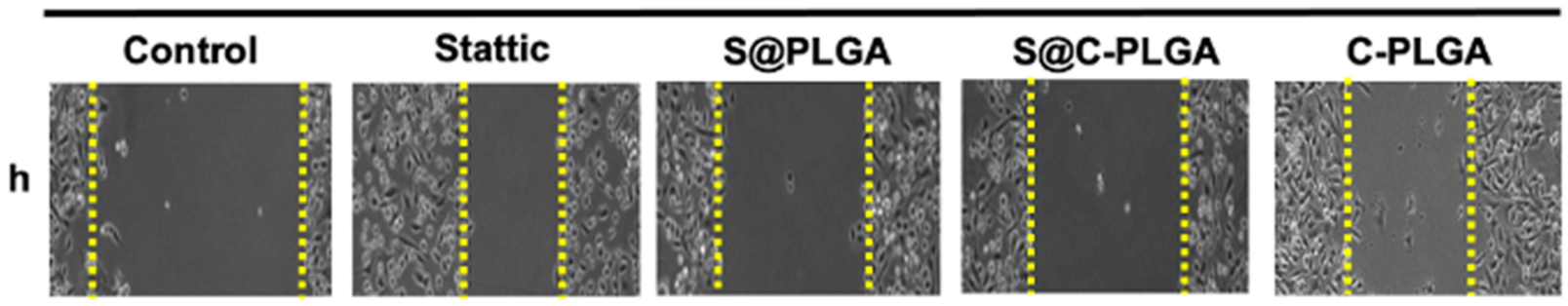

$12 \mathrm{~h}$
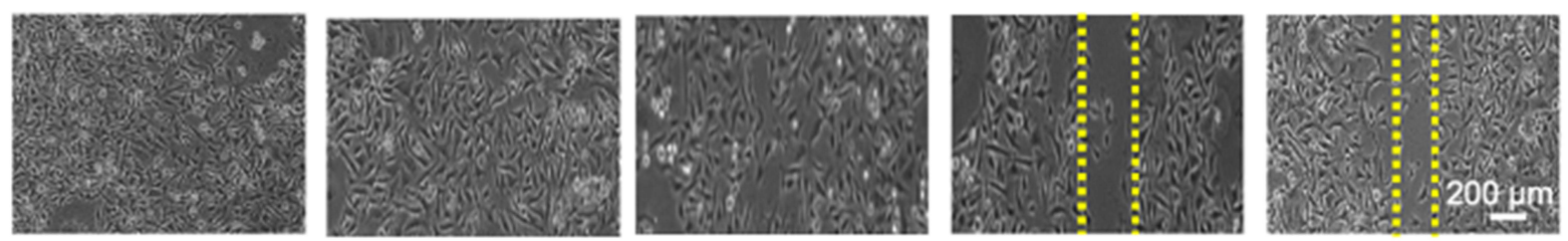

B

4T1
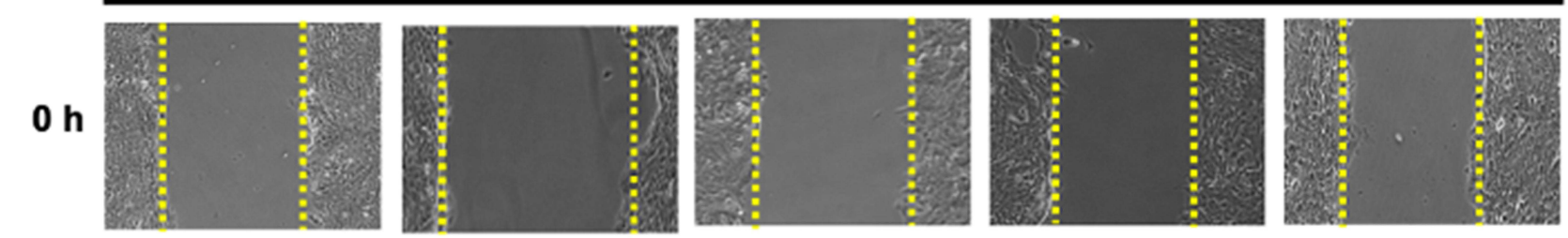

$12 \mathrm{~h}$
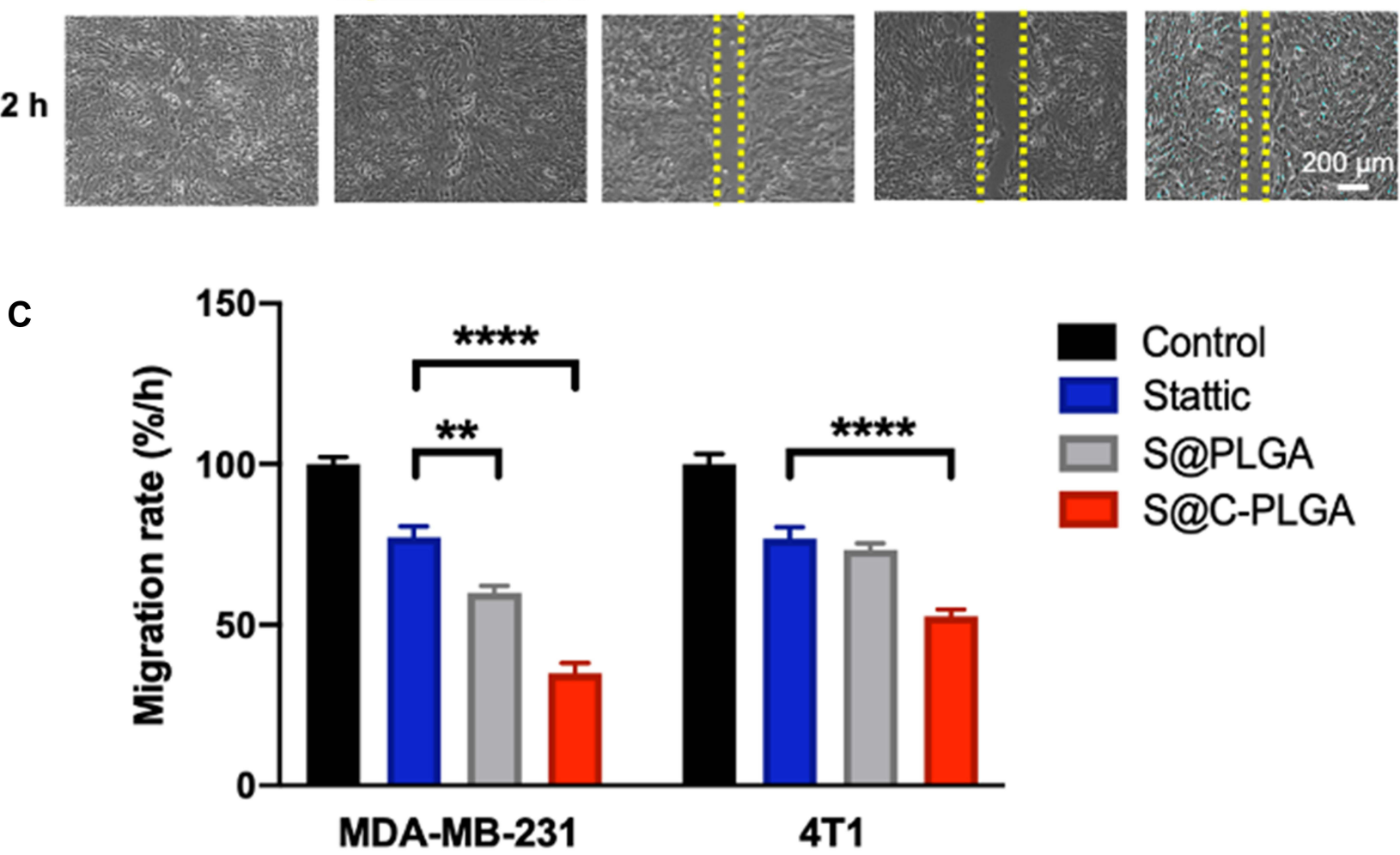

Figure 3 In vitro anti-migration properties of Stattic, S@PLGA and S@C-PLGA NPs in Scratch Assay. Microscopic images of the Scratch Assay on MDA-MB-23I (A) and 4TI (B) at different time points. (C) The normalized migration rate of MDA-MB-23I and 4TI cells after being treated with Stattic, S@PLGA and S@C-PLGA NPs at I $\mu$ M Stattic equivalent concentration for $24 \mathrm{~h}$. Data represent mean \pm SEM from three independent experiments; $* *$ and $* * * *$ Indicate $\mathrm{p}<0.0 \mathrm{I}, 0.000 \mathrm{I}$, respectively, as assessed by two-way ANOVA with Tukey's post-hoc test.

NPs. The findings on the lower in vitro potency of Stattic were consistent with those reported by Han et al, where a high concentration $(10 \mu \mathrm{M})$ of Stattic was required to downregulate the expression of metastasis signaling proteins such as c-Myc and survivin. Meanwhile, the observed increase in antimigration potency is likely due 
to the increase in cellular uptake of S@C-PLGA relative to S@PLGA and Stattic, as corroborated by our earlier work on chitosan-coated PLGA-BODIPY. ${ }^{17}$ The increased cellular uptake of S@C-PLGA may also contribute to the mucoadhesive behavior of chitosan, which is able to increase penetration through the mucus layer. ${ }^{38}$ Similar trends of cell migration inhibition were observed in the transwell assay, whereby the S@C-PLGA exhibited better antimigration activity than Stattic and non-coated S@PLGA at $1 \mu \mathrm{M}$ Stattic equivalent concentration (Figure 4).

In our previous work, C-PLGA NPs loaded with BODIPY exhibited better in vivo tumor-targeting selectivity and reduced accumulation in RES tissue and non-tumorous organs, as well as reduced protein adsorption and macrophage uptake compared with uncoated PLGA NPs. ${ }^{17}$ Since S@C-PLGA was shown to have a better in vitro antimigration effect and better drug release profile in this study, only the S@C-PLGA NPs were subjected to in vivo antitumor and antimetastatic effects.

\section{Preliminary in vivo Study}

In vivo Toxicity Profiling

Stattic and S@C-PLGA NPs were administered to mice via the tail vein at doses of up to $24 \mathrm{mg}$ Stattic or Stattic equivalent (S@C-PLGA)/kg (200 mg/kg of
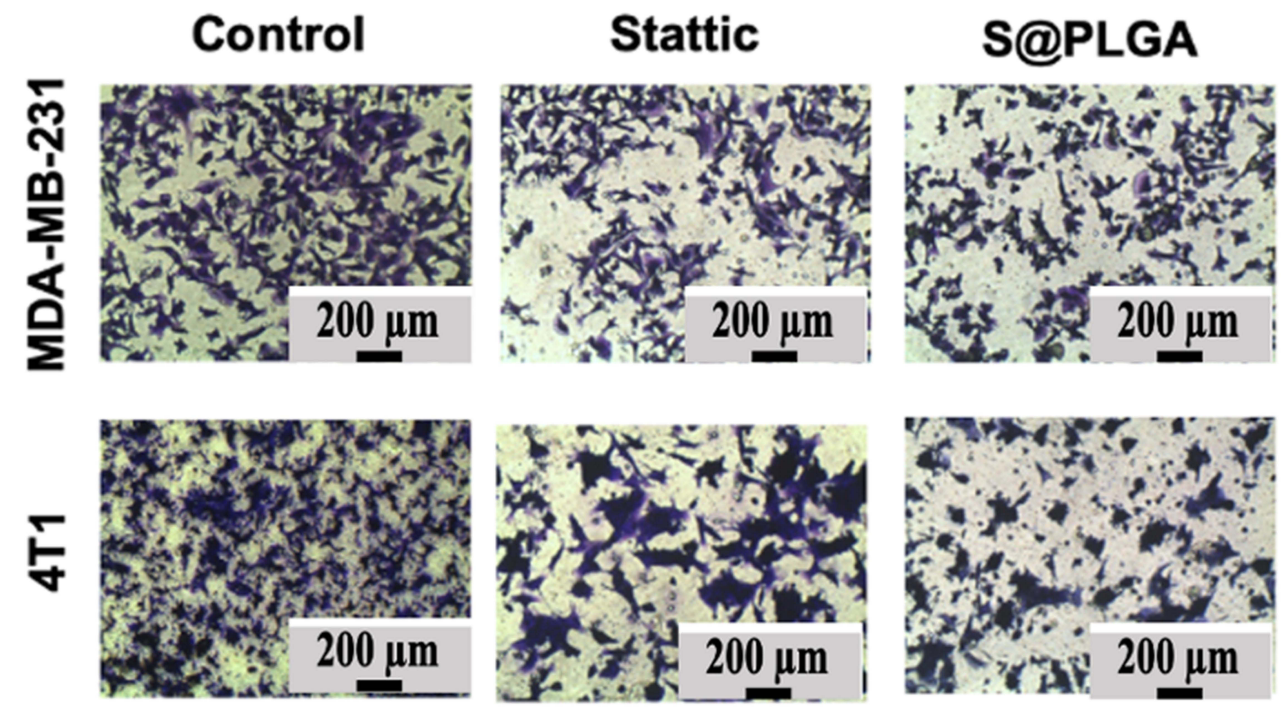

\section{S@C-PLGA}
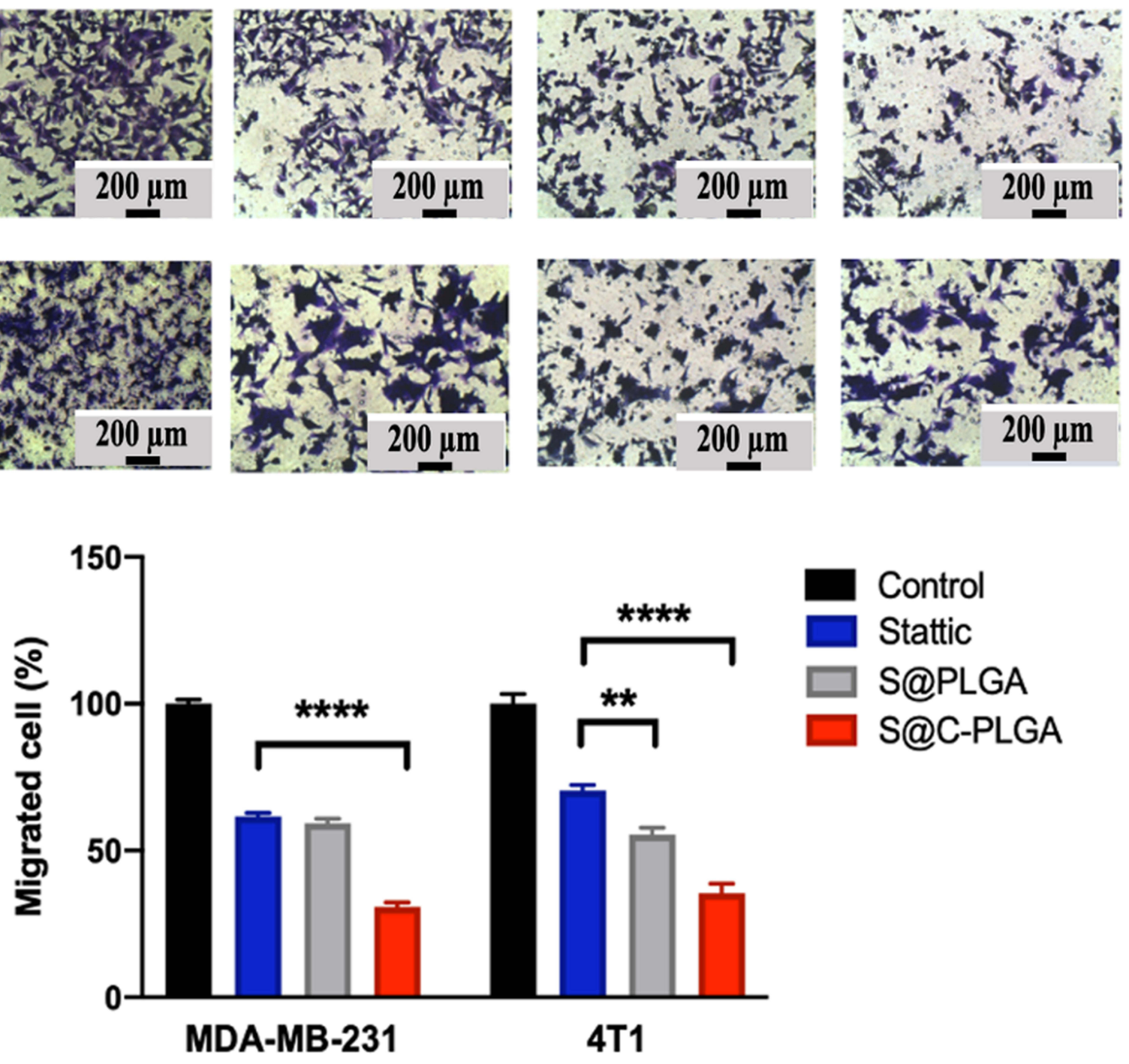

Figure 4 In vitro anti-migration properties of Stattic, S@PLGA and S@C-PLGA NPs in transwell assay. Microscopic images of the transwell migration assay in MDA-MB-23I and 4TI after treated with I $\mu$ M of Stattic, S@PLGA NPs and S@C-PLGA NPs. The percentage of migrated cells relative to control were quantified using Imagej software version 2.0. Data represent mean \pm SEM from three independent experiments; $* *$ and $* * * *$ Indicate $p<0.01,0.000$ I, respectively, as assessed by two-way ANOVA with Tukey's post-hoc test. 
S@C-PLGA). The mice were then monitored for 14 days for weight loss, death, and signs of toxicity such as inactivity, ruffled fur, and behavioral changes, according to the specifications of the Berlin test for in vivo toxicity assessment. ${ }^{17}$ No death, significant changes in body weight (Figure 5A), organ histology (Figure S6), or other signs of toxicity were observed in mice receiving Stattic or S@C-PLGA NPs at the highest Stattic/ Stattic equivalent dose tested.

\section{In vivo Tumor Growth Attenuation Study}

BALB/c mice bearing 4T1 mammary tumors approximately $70 \mathrm{~mm}^{3}$ in size were treated with four consecutive doses (on days 0, 3, 6, and 9) of S@C-PLGA NPs (24 mg Stattic equivalent $/ \mathrm{kg})$, Stattic ( $24 \mathrm{mg} / \mathrm{kg})$, and normal saline (negative control group). The antitumor effects (tumor growth suppression) and antimetastatic effects (histological assessments) were assessed.

Multiple intravenous administration of Stattic reduced the area under the curve (AUC) by $31.6 \%$ as compared with the saline control $(\mathrm{p}<0.01$, one-way ANOVA, Dunnett's test; Figure 5B). It has been reported that activation of STAT3 induces M2 macrophage polarization, which promotes tumor growth and angiogenesis. ${ }^{39,40}$ As Stattic inhibits STAT3 activation, M2 polarization is likely to decrease in Stattic treated mice, thereby suppressing tumor growth. ${ }^{41,42}$

On the other hand, a higher degree of tumor growth suppression was observed in mice that received multiple doses of S@C-PLGA NPs (24 mg Stattic equivalent /kg) with a 54.6\% reduction in AUC compared with the saline group ( $\mathrm{p}<0.0001)$. The tumor growth suppression effect of Stattic entrapped within C-PLGA was enhanced by $33.6 \%$, based on AUC, when compared with that of the Stattic only $(p<0.01)$. The enhanced tumor growth suppression efficacy of S@C-PLGA could be ascribed to the higher passive accumulation of nanocarriers in tumors through the EPR effect, as corroborated by our previous study, where chitosan-coated PLGA NPs prolonged the accumulation of photosensitizer in tumors. ${ }^{17}$

Although Stattic's tumor growth suppression efficacy was enhanced by entrapment into the C-PLGA nanocarriers, complete tumor suppression was not achieved. This result was consistent with a previous study, where incomplete tumor suppression was observed in Stattic-treated mice. $^{43}$ Stattic is commonly used as an antimetastatic agent or sensitizer for radio- and chemotherapy. $7,9,43,44$ More importantly, the observation of improved tumor growth suppression efficacy in mice receiving S@C-PLGA has indicated an increased accumulation of Stattic in the primary tumor, which is crucial for the exertion of Stattic's antimetastatic effects.

\section{In vivo Antimetastatic Effect of S@C-PLGA NPs}

The in vivo antimetastatic effect of S@C-PLGA was investigated by assessing the histology (H\&E staining) of the major organs (liver, lung, kidney, heart, and lymph nodes) harvested from the healthy mice (Figure 6A) and $4 \mathrm{~T} 1$ tumor-bearing mice at the end of the multiple-dose treatment studies (Figure 6B-D). The number of
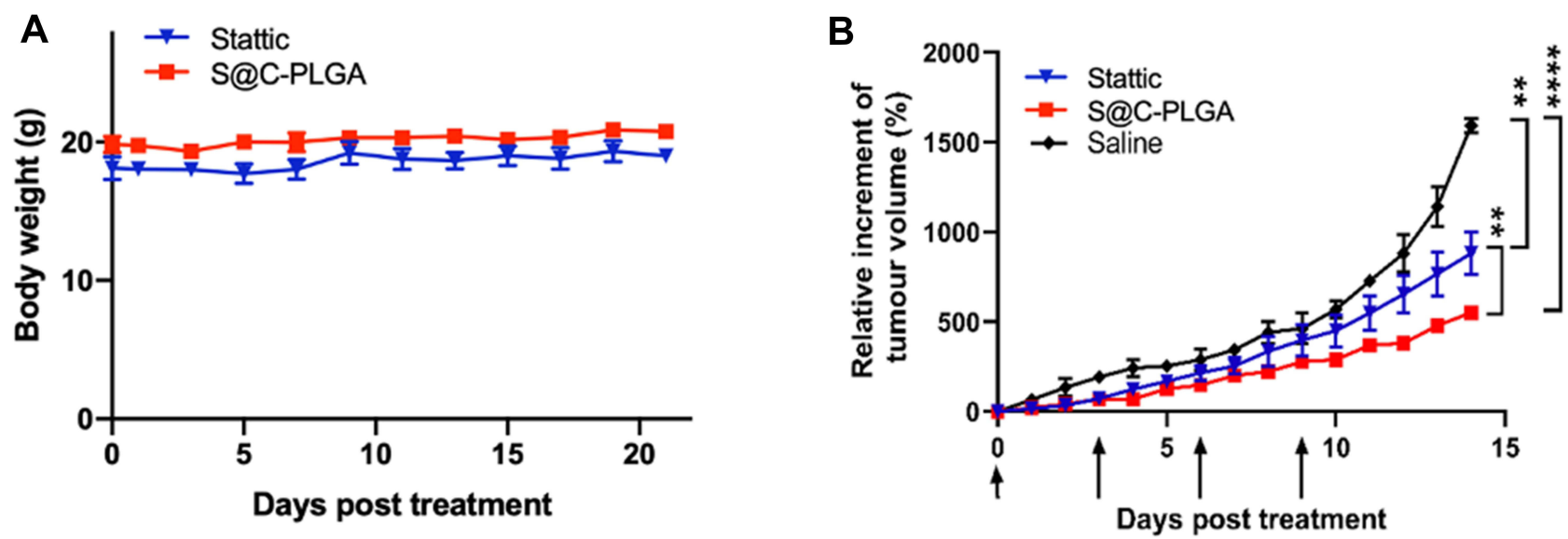

Figure 5 (A) In vivo toxicity profiling and antitumor efficacy study of Stattic and S@C-PLGA NPs. In vivo toxicity profile of Stattic and S@C-PLGA NPs in 4TI tumorbearing mice. Data represents the mean body weights \pm SEM $(n=3)$ for each group. $(B)$ In vivo antitumor efficacy of Stattic and S@C-PLGA NPs in 4TI tumor-bearing mice. S@C-PLGA NPs (24 mg Stattic eqv./kg) showed greater suppression in 4TI tumor growth compared to Stattic $(24 \mathrm{mg} / \mathrm{kg})$ and saline. The day of treatment was indicated as black arrows. Data represents the mean tumor volume \pm SEM $(n=4)$ for each group; ** and **** indicate $p<0.01,0.000$, respectively, as assessed by one-way ANOVA with Dunnett's post-hoc test. 

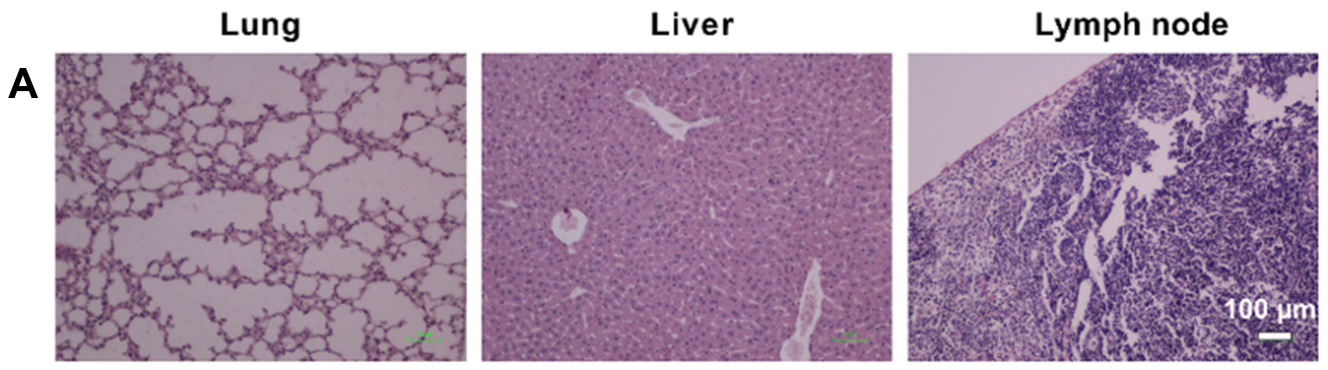

B
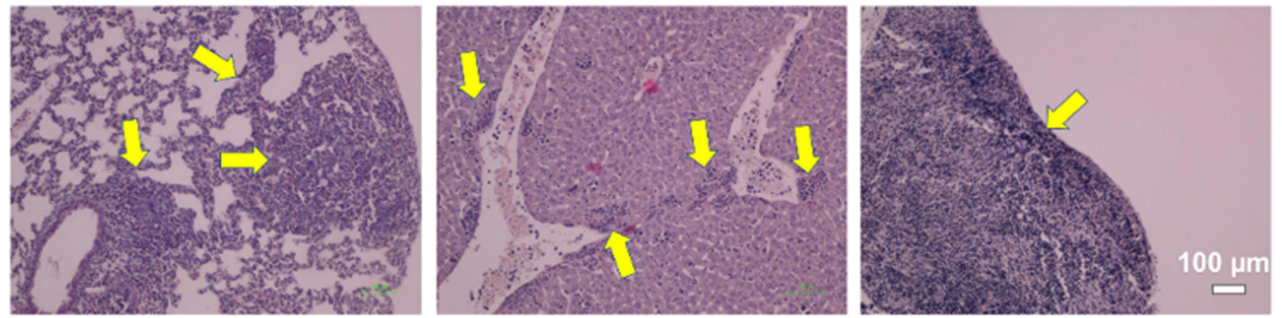

C
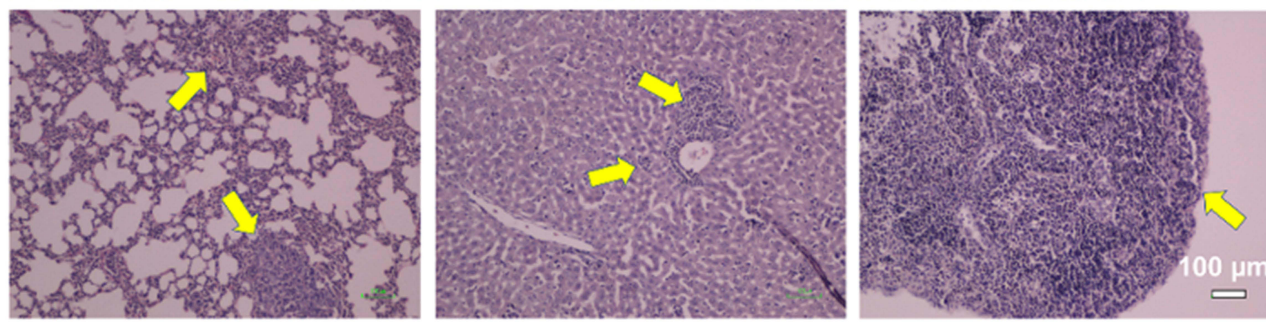

D
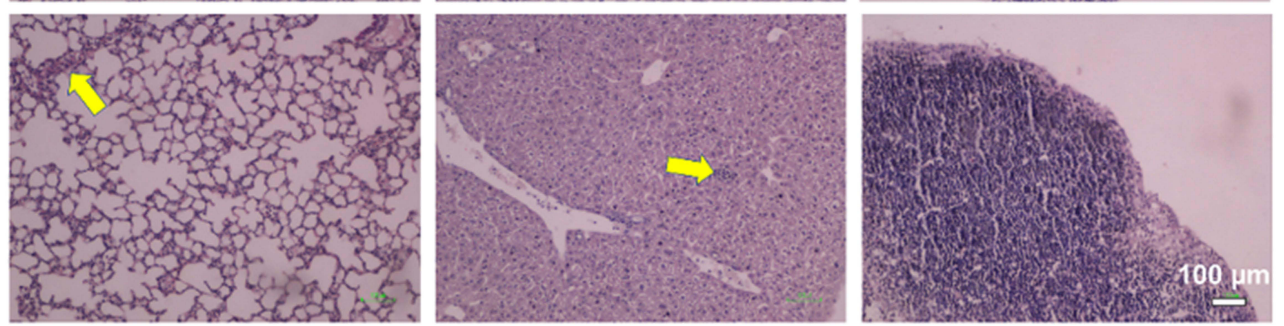

$\mathbf{E}$

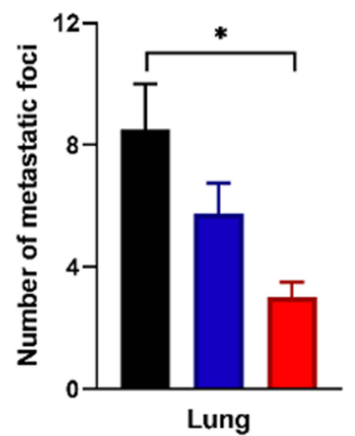

$\mathbf{F}$

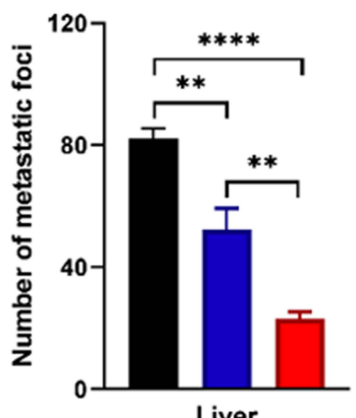

G

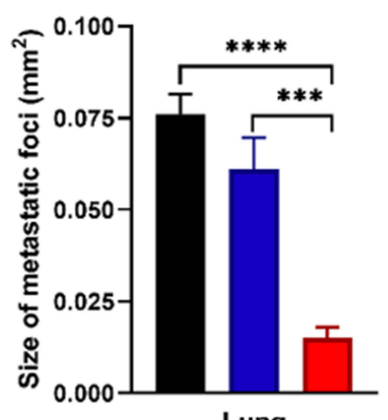

Lung
H

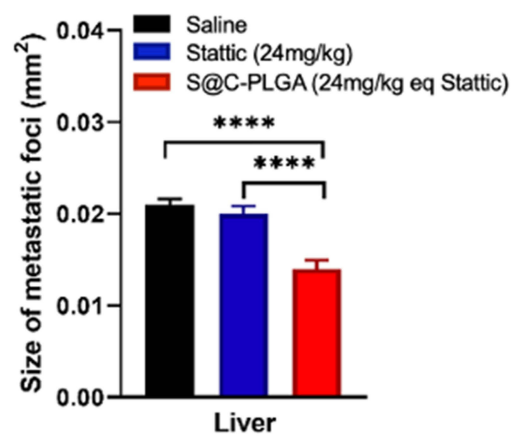

Figure 6 In vivo antimetastatic effects of S@C-PLGA NPs. Histopathological analysis and quantification of metastatic foci for lung, liver, and lymph node of untreated control mice (A) and 4TI tumor-bearing mice treated with multiple doses of saline (B), Stattic (24 mg/kg) (C) and S@C-PLGA NPs (24 mg Stattic eqv./kg) (D). No metastatic foci were observed in heart, kidney and spleen. Quantification of number of metastatic foci found in lung (E) and liver (F) by observing 5 and 8 images in lung and liver, respectively, for each mouse in all the treatment groups. Average sizes of metastatic foci found in lung $(\mathbf{G})$ and liver $(\mathbf{H})$ for each treatment group. Data represent the mean number of metastatic foci \pm SEM $(n=4) ; *$, **, *** and ****Indicate $\mathrm{p}<0.05,0.0 \mathrm{I}, 0.00 \mathrm{I}$ and 0.000 I, respectively as assessed by One-way ANOVA with Dunnett's post-hoc test. Yellow arrows indicate metastatic foci.

metastatic foci found on the prepared tissue slides was counted (Figure 6E and F), and the area of metastatic foci was measured using the Image $\mathrm{J}$ software (Figure 6G and $\mathrm{H}$ ).
Large pulmonary metastatic foci $(9 \pm 2$ foci, average foci area at $0.076 \pm 0.005 \mathrm{~mm}^{2}$; Figure $6 \mathrm{~B}$ and $\mathrm{E}$ ), and multiple liver micrometastases ( $82 \pm 3$ foci; Figure $6 \mathrm{~B}$ and F) were found in tumor-bearing mice that received normal 
saline. Both pulmonary and hepatic metastases are commonly found in 4T1 orthotropic mammary tumor metastasis models. ${ }^{45}$ Stattic-treated 4T1 tumor-bearing mice exhibited a mild antimetastatic effect. The numbers of metastatic foci in the lungs ( $6 \pm 1$ foci) and livers (53 \pm 7 foci) were reduced by $33.3 \%$ and $35.4 \%(\mathrm{p}<0.01)$, respectively, compared with saline-treated mice (one-way ANOVA followed by Dunnett's test). The average size of the pulmonary metastatic foci of Stattic-treated mice was also reduced by $19.7 \%$ (average foci area at $0.061 \pm 0.009 \mathrm{~mm}^{2}$ ) compared with the saline-treated group. No significant change was noted in the size of the liver metastatic foci compared with the saline control group (Figure 6G and $\mathrm{H}$ ).

Interestingly, entrapment of Stattic into the C-PLGA nanocarrier (S@C-PLGA) significantly enhanced its antimetastatic effect in 4T1 tumor-bearing mice. Greater reductions in the numbers of metastatic foci in the lungs $(3 \pm 1$ foci) and livers ( $23 \pm 2$ foci) were observed (66.7\% and $72.0 \%$ reduction, $\mathrm{p}<0.05$ and $\mathrm{p}<0.0001$, respectively) in the S@C-PLGA-treated 4T1 tumor-bearing mice compared with the saline control. These values were $50.0 \%$ and $56.6 \%$ lower than the average foci count found in the lungs and livers of Stattic-treated 4T1 tumor-bearing mice. S@C-PLGA treatment inhibited the formation of large pulmonary metastases in mice, whereby the sizes of pulmonary metastatic foci (average foci area at $0.015 \pm 0.003 \mathrm{~mm}^{2}$ ) were reduced significantly (by $80.3 \%$ and $75.4 \%$, respectively) compared with those in mice receiving saline and Stattic ( $p<0.0001$ and $p<0.001$; Figure $6 D$ and $G$ ). A lower degree of metastasis to the lymph node was also seen in S@C-PLGA-treated mice than in Stattic-treated mice. The improved in vivo metastasis suppression by S@C-PLGA may be attributed to the increase in its accumulation in the mouse primary tumor and potentially in the newly formed metastatic foci with high angiogenesis activity, ${ }^{46}$ as reflected by the tumor growth delay following S@C-PLGA treatment and the reduction in the number and size of newly formed metastatic foci. ${ }^{17,20,47}$

Extending the progression-free survival period of cancer patients has become a primary aim for cancer therapy and palliative care, and a requirement for new drug approvals in oncology by the US Food and Drug Administration (FDA). ${ }^{48}$ Although antimetastatic agents may extend the progression-free survival period in patients with metastatic disease, adverse effects have been correlated to the random drug dissemination and use that is limited to a "dosed until unacceptable toxicity" basis in clinical settings. ${ }^{49,50}$ Nanocarriers have been frequently used to reduce random drug dissemination in the body and to improve tumortargeted delivery of cytotoxic drugs. ${ }^{51}$ In contrast, studies in the nano-delivery of small molecular antimetastatic drugs are relatively few. ${ }^{52}$ In view of the recent uptrend in using small molecular antimetastatic agents for the extension of progression-free survival period and improvement of survival outcomes of cancer patients, further research on the nano-delivery of small molecular antimetastatic drugs for toxicity reduction and efficacy enhancement may become increasingly important. The current study has illustrated the use of C-PLGA nanocarriers to improve the in vitro and in vivo antimetastatic activity of Stattic and hence may be a potential adjunctive antimetastatic agent for cancer therapy.

\section{Conclusions}

This study demonstrates the improvement in the antimigration potency of Stattic upon its entrapment into the C-PLGA nanocarrier. S@C-PLGA showed a predominant effect as an antimetastatic agent in vivo. Although the 4T1 tumor was not completely suppressed by the Stattic entrapped within C-PLGA owing to the antimetastatic (not cytotoxic) nature of Stattic, the in vivo metastasis of 4T1 cells was effectively suppressed, as demonstrated by the reduction in the numbers of metastatic foci in the lungs $(50.0 \%)$ and livers $(56.6 \%)$, as well as in the size $(75.4 \%)$ of the lung metastatic foci of mice that received S@C-PLGA when compared with that of mice that received Stattic. These results suggest the potential of employing nanocarriers to improve the delivery and efficacy of small molecular antimetastatic drugs.

\section{Acknowledgments}

This work is supported by the Fundamental Research Grant Scheme (FRGS/1/2017/SKK10/UM/02/1) from the Ministry of Higher Education, Malaysia, and MOST grants of Taiwan (MOST 107-2112-M-009-016-MY3, MOST 109-2811-M-009-502, MOST 109-2927-I-009003, MOST 110-2112-M-A49-005, and MOST 110-2927I-A49-003) and from Ministry of Education, Taiwan through the SPROUT Project-Center for Intelligent Drug Systems and Smart Biodevices $\left(\operatorname{IDS}^{2} \mathrm{~B}\right)$ of NYCU, Taiwan.

\section{Disclosure}

The authors report no conflicts of interest in this work. 


\section{References}

1. Steeg PS. Targeting metastasis. Nat Rev Cancer. 2016;16 (4):201-218. doi:10.1038/nrc.2016.25

2. Ma J, Kala S, Yung S, et al. Blocking stemness and metastatic properties of ovarian cancer cells by targeting p70(S6K) with dendrimer nanovector-based sirna delivery. Mol Ther. 2018;26(1):70-83. doi:10.1016/j.ymthe.2017.11.006

3. Gong N, Teng X, Li J, Liang XJ. Antisense oligonucleotide-conjugated nanostructure-targeting lncrna MALAT1 inhibits cancer metastasis ACS Appl Mater Interfaces. 2019;11(1):37-42. doi:10.1021/ acsami.8b18288

4. Yang CL, Chao YJ, Wang HC, et al. Local ablation of gastric cancer by reconstituted apolipoprotein B lipoparticles carrying epigenetic drugs. Nanomedicine. 2021;37:102450. doi:10.1016/j.nano.2021.102450

5. Chu HL, Cheng TM, Chen HW, et al. Synthesis of apolipoprotein B lipoparticles to deliver hydrophobic/amphiphilic materials. ACS Appl Mater Interfaces. 2013;5(15):7509-7516. doi:10.1021/am401808e

6. Hanafy NAN, Quarta A, Ferraro MM, et al. Polymeric nano-micelles as novel cargo-carriers for Ly2157299 liver cancer cells delivery. Int J Mol Sci. 2018;19(3):748-760. doi:10.3390/ijms 19030748

7. Chiba T. STAT3 inhibitors for cancer therapy - the rationale and remained problems. EC Cancer. 2016;1:S1-S8.

8. Kim MS, Lee WS, Jeong J, Kim SJ, Jin W. Induction of metastatic potential by trkb via activation of IL6/JAK2/STAT3 and PI3K/AKT signaling in breast cancer. Oncotarget. 2015;6(37):40158-40171. doi:10.18632/oncotarget.5522

9. Pan Y, Zhou F, Zhang R, Claret FX. Stat3 inhibitor stattic exhibits potent antitumor activity and induces chemo- and radio-sensitivity in nasopharyngeal carcinoma. PLoS One. 2013;8(1):e54565. doi:10. 1371/journal.pone. 0054565

10. Schust J, Sperl B, Hollis A, Mayer TU, Berg T. Stattic: a small-molecule inhibitor of Stat3 activation and dimerization. Chem Biol. 2006;13(11):1235-1242. doi:10.1016/j.chembiol.2006.09.018

11. Yue P, Turkson J. Targeting STAT3 in cancer: how successful are we? Expert Opin Investig Drugs. 2009;18(1):45-56. doi:10.1517/ 13543780802565791

12. Bohrer LR, Chuntova P, Bade LK, et al. Activation of the FGFR-STAT3 pathway in breast cancer cells induces a hyaluronan-rich microenvironment that licenses tumor formation. Cancer Res. 2014;74(1):374-386. doi:10.1158/0008-5472.CAN-13-2469

13. Vicent MJ, Duncan R. Polymer conjugates: nanosized medicines for treating cancer. Trends Biotechnol. 2006;24(1):39-47. doi:10.1016/j. tibtech.2005.11.006

14. Hanafy NAN, Quarta A, Di Corato R, et al. Hybrid polymeric-protein nano-carriers (HPPNC) for targeted delivery of TGF $\beta$ inhibitors to hepatocellular carcinoma cells. Mater Sci Mater Med. 2017;28 (8):120-130. doi:10.1007/s10856-017-5930-7

15. Hu Q, Sun W, Wang C, Gu Z. Recent advances of cocktail chemotherapy by combination drug delivery systems. Adv Drug Deliv Rev. 2016;98:19-34. doi:10.1016/j.addr.2015.10.022

16. Maeda H, Nakamura H, Fang J. The EPR effect for macromolecular drug delivery to solid tumors: improvement of tumor uptake, lowering of systemic toxicity, and distinct tumor imaging in vivo. $A d v$ Drug Deliv Rev. 2013;65(1):71-79. doi:10.1016/j.addr.2012.10.002

17. Voon SH, Tiew SX, Kue CS, et al. Chitosan-coated poly(lactic-coglycolic acid)-diiodinated boron-dipyrromethene nanoparticles improve tumor selectivity and stealth properties in photodynamic cancer therapy. J Biomed Nanotechnol. 2016;12(7):1431-1452. doi:10.1166/jbn.2016.2263

18. Tiew SX, Misni M. Thermal properties of acylated low molecular weight chitosans. J Chem Soc Pak. 2019;41:207-218.

19. Fessi H, Puisieux F, Devissaguet JP, Ammoury N, Benita S. Nanocapsule formation by interfacial polymer deposition following solvent displacement. Int J Pharm. 1989;55(1):R1-R4. doi:10.1016/ 0378-5173(89)90281-0
20. Kefayat A, Vaezifar S. Biodegradable PLGA implants containing doxorubicin-loaded chitosan nanoparticles for treatment of breast tumor-bearing mice. Int J Biol Macromol. 2019;136:48-56. doi:10.1016/j.ijbiomac.2019.06.055

21. Liang CC, Park AY, Guan JL. In vitro scratch assay: a convenient and inexpensive method for analysis of cell migration in vitro. Nat Protoc. 2007;2(2):329-333. doi:10.1038/nprot.2007.30

22. Anasamy T, Thy CK, Lo KM, et al. Tribenzyltin carboxylates as anticancer drug candidates: effect on the cytotoxicity, motility and invasiveness of breast cancer cell lines. Eur J Med Chem. 2017;125:770-783. doi:10.1016/j.ejmech.2016.09.061

23. Holen I, Lawson MA. Chapter 43 - In vivo models used in studies of bone metastases. In: Heymann D, editor. Bone Cancer. 2nd ed. San Diego: Academic Press; 2015:503-518.

24. Delgado-SanMartin J, Ehrhardt B, Paczkowski M, et al. An innovative non-invasive technique for subcutaneous tumour measurements. PLoS One. 2019;14(10):e0216690. doi:10.1371/ journal.pone. 0216690

25. Faustino-Rocha A, Oliveira PA, Pinho-Oliveira J, et al. Estimation of rat mammary tumor volume using caliper and ultrasonography measurements. Lab Anim. 2013;42(6):217-224. doi:10.1038/laban.254

26. Foo YY, Periasamy V, Kiew LV, Kumar GG, Malek SNA. Curcuma mangga-mediated synthesis of gold nanoparticles: characterization, stability, cytotoxicity, and blood compatibility. Nanomaterials. 2017;7(6):1-14. doi:10.3390/nano7060123

27. Fissan H, Ristig S, Kaminski H, Asbach C, Epple M. Comparison of different characterization methods for nanoparticle dispersions before and after aerosolization. Anal Methods. 2014;6(18):7324-7334. doi:10.1039/C4AY01203H

28. Blanco E, Shen H, Ferrari M. Principles of nanoparticle design for overcoming biological barriers to drug delivery. Nat Biotechnol. 2015;33(9):941-951. doi:10.1038/nbt.3330

29. Wang Q, Tan L, Yang K. Cytocompatibility and hemolysis of AZ31B magnesium alloy with Si-containing coating. J Mater Sci Technol. 2015;31(8):845-851. doi:10.1016/j.jmst.2015.07.008

30. Badran MM, Alomrani AH, Harisa GI, Ashour AE, Kumar A, Yassin AE. Novel docetaxel chitosan-coated PLGA/PCL nanoparticles with magnified cytotoxicity and bioavailability. Biomed Pharmacother. 2018;106:1461-1468. doi:10.1016/j.biopha.2018.07.102

31. Affandi ISM, Lee WQ, Feroz SR, Mohamad SB, Tayyab S. Interaction of stattic, a STAT3 inhibitor with human serum albumin: spectroscopic and computational study. J Biomol Struct Dyn. 2017;35 (16):3581-3590. doi:10.1080/07391102.2016.1264887

32. Viswanathan G, Hsu YH, Voon SH, et al. A comparative study of cellular uptake and subcellular localization of doxorubicin loaded in self-assemblies of amphiphilic copolymers with pendant dendron by MDA-MB-231 human breast cancer cells. Macromol Biosci. 2016;16 (6):882-895. doi:10.1002/mabi.201500435

33. Kwa YC, Tan YF, Foo YY, et al. Improved delivery and antimetastatic effects of Stattic by self-assembled amphiphilic pendant-dendron copolymer micelles in breast cancer cell lines. J Drug Deliv Sci Technol. 2020;59:101905-101916. doi:10.1016/j.jddst.2020.101905

34. Noori Koopaei M, Khoshayand MR, Mostafavi SH, et al. Docetaxel loaded PEG-PLGA nanoparticles: optimized drug loading, in-vitro cytotoxicity and in-vivo antitumor effect. Iran J Pharm Res. 2014;13 (3):819-833.

35. Palocci C, Valletta A, Chronopoulou L, et al. Endocytic pathways involved in PLGA nanoparticle uptake by grapevine cells and role of cell wall and membrane in size selection. Plant Cell Rep. 2017;36 (12):1917-1928. doi:10.1007/s00299-017-2206-0

36. Welch DR, Hurst DR. Defining the hallmarks of metastasis. Cancer Res. 2019;79(12):3011-3027. doi:10.1158/0008-5472.CAN-19-0458

37. Liu Y-L, Chou C-K, Kim M, et al. Assessing metastatic potential of breast cancer cells based on EGFR dynamics. Sci Rep. 2019;9 (1):3395-3407. doi:10.1038/s41598-018-37625-0 
38. Hanafy NAN. Optimally designed theranostic system based folic acids and chitosan as a promising mucoadhesive delivery system for encapsulating curcumin LbL nano-template against invasiveness of breast cancer. Int J Biol Macromol. 2021;182:1981-1993. doi:10.1016/j.ijbiomac.2021.05.149

39. Mu X, Shi W, Xu Y, et al. Tumor-derived lactate induces M2 macrophage polarization via the activation of the ERK/STAT3 signaling pathway in breast cancer. Cell Cycle. 2018;17(4):428-438. doi: $10.1080 / 15384101.2018 .1444305$

40. Jayasingam SD, Citartan M, Thang TH, Mat Zin AA, Ang KC, Ch'ng ES. Evaluating the polarization of tumor-associated macrophages into $\mathrm{m} 1$ and $\mathrm{m} 2$ phenotypes in human cancer tissue: technicalities and challenges in routine clinical practice. Front Oncol. 2020;9:1512. doi:10.3389/fonc.2019.01512

41. Lv R, Bao Q, Li Y. Regulation of M1-type and M2-type macrophage polarization in RAW264.7 cells by Galectin-9. Mol Med Rep. 2017;16(6):9111-9119. doi:10.3892/mmr.2017.7719

42. Yin Z, Ma T, Lin Y, et al. IL-6/STAT3 pathway intermediates M1/M2 macrophage polarization during the development of hepatocellular carcinoma. J Cell Biochem. 2018;119(11):9419-9432. doi:10.1002/ jcb. 27259

43. Liu Z, Ge X, Gu Y, et al. Small molecule STAT3 inhibitor, 6Br-6a suppresses breast cancer growth in vitro and in vivo. Biomed Pharmacother. 2020;121:109502-109510. doi:10.1016/j.biopha.20 19.109502

44. Adachi M, Cui C, Dodge CT, Bhayani MK, Lai SY. Targeting STAT3 inhibits growth and enhances radiosensitivity in head and neck squamous cell carcinoma. Oral Oncol. 2012;48(12):1220-1226. doi:10.1016/j.oraloncology.2012.06.006
45. Gao D, Du J, Cong L, Liu Q. Risk factors for initial lung metastasis from breast invasive ductal carcinoma in stages I-III of operable patients. Jpn J Clin Oncol. 2008;39(2):97-104. doi:10.1093/jjco/ hyn 133

46. Ross MH, Esser AK, Fox GC, et al. Bone-induced expression of integrin $\beta 3$ enables targeted nanotherapy of breast cancer metastases. Cancer Res. 2017;77(22):6299-6312. doi:10.1158/0008-5472.CAN17-1225

47. Lee SY, Koo JS, Yang M, Cho HJ. Application of temporary agglomeration of chitosan-coated nanoparticles for the treatment of lung metastasis of melanoma. J Colloid Interface Sci. 2019;544:266-275. doi:10.1016/j.jcis.2019.02.092

48. Hess LM, Brnabic A, Mason O, Lee P, Barker S. Relationship between progression-free survival and overall survival in randomized clinical trials of targeted and biologic agents in oncology. $J$ Cancer. 2019;10(16):3717-3727. doi:10.7150/jca.32205

49. Murthy RK, Loi S, Okines A, et al. Tucatinib, trastuzumab, and capecitabine for HER2-positive metastatic breast cancer. $N$ Engl $J$ Med. 2020;382(7):597-609. doi:10.1056/nejmoa1914609

50. Geyer CE, Forster J, Lindquist D, et al. Lapatinib plus capecitabine for HER2-positive advanced breast cancer. $N$ Engl J Med. 2006;355 (26):2733-2743. doi:10.1056/nejmoa064320

51. Saw WS, Anasamy T, Foo YY, et al. Delivery of nanoconstructs in cancer therapy: challenges and therapeutic opportunities. Adv Ther. 2021;4(3):2000206. doi:10.1002/adtp.202000206.

52. Gonciar D, Mocan T, Matea CT, et al. Nanotechnology in metastatic cancer treatment: current achievements and future research trends. $J$ Cancer. 2019;10(6):1358-1369. doi:10.7150/jca.28394
International Journal of Nanomedicine

\section{Publish your work in this journal}

The International Journal of Nanomedicine is an international, peerreviewed journal focusing on the application of nanotechnology in diagnostics, therapeutics, and drug delivery systems throughout the biomedical field. This journal is indexed on PubMed Central, MedLine, CAS, SciSearch ${ }^{\mathbb{}}$, Current Contents ${ }^{\mathbb{R}} /$ Clinical Medicine, $^{-}$

\section{Dovepress}

Journal Citation Reports/Science Edition, EMBase, Scopus and the Elsevier Bibliographic databases. The manuscript management system is completely online and includes a very quick and fair peer-review system, which is all easy to use. Visit http://www.dovepress.com/ testimonials.php to read real quotes from published authors. 